\title{
Intrathoracic stomach mimicking bone metastasis from thyroid cancer in whole-body iodine-131 scan diagnosed by SPECT/CT
}

Francisco Javier García-Gómez', Pablo Antonio de la Riva-Pérez', Cinta Calvo-Morón', Cristina Buján-Lloret', Teresa Cambil-Molina', Juan Castro-Montaño'

\section{SUMMARY}

The whole-body iodine-131 scintigraphy is an imaging technique in monitoring patients with a history of thyroid cancer. Although the rate of false positives is negligible, it is not nonexistent. We report the case of an intervened and treated patient for thyroid cancer with good clinical and biochemical response. Scintigraphic findings were consistent with unsuspected bone metastasis. Fused SPECT/CT data allowed accurate diagnosis of giant diaphragmatic hernia associated with intrathoracic stomach, a very rare pathology that can lead to false positive results. Arch Endocrinol Metab. 2017;61(3):288-90.

1 Department of Nuclear Medicine, Virgen Macarena University Hospital, Sevilla, Spain

Correspondence to: Francisco Javier García-Gómez javier191185@gmail.com

Received on Jan/8/2016 Accepted on Oct/18/2016

DOI: 10.1590/2359-39970000000243

\section{CASE REPORT}

$\mathrm{W}$ e report a 76 year old female with history of TIN0M0 well differentiated papillary thyroid cancer being operated and treated with ablative dose of iodine-131 $\left.{ }^{(131} \mathrm{I}\right)$. During regular followup, a whole-body ${ }^{131}$ I scintigraphy was performed 12 months after treatment ends in order to evaluate the persistence of thyroid remainders. Whole-body ${ }^{131} \mathrm{I}$ scan (Figure 1) revealed high intense hypermetabolic uptake foci, located in the midline. This finding was observed with greater intensity of uptake in the posterior projection (Figure 1, right panel; black arrow) being consistent with disease progression as spine bone metastasis. Intense back pain was referred when the patient was purposely questioned. Hormone levels at moment of scan were stimulated $\mathrm{Tg}<0.2 \mathrm{ngr} / \mathrm{mL}$, nonstimulated $\mathrm{Tg}<0.2 \mathrm{ngr} / \mathrm{mL}$ and antithyroglobulin antibodies (TgAB) 349.4 U/L (Reference range: 0.0$200.0 \mathrm{U} / \mathrm{L})$.

Subsequently, a single photon emission computed tomography/computed tomography (SPECT/CT) was performed due to discordance between biochemical findings (low hormone levels) and the clinical picture of intense back pain and functional imaging consistent with spine bone metastasis. A severe thoracolumbar scoliosis that determines a giant diaphragmatic hernia was revealed thanks to multimodality scan (Figure 2). Thereby, high intense hypermetabolic foci corresponded to physiological activity of the improperly positioned gastric mucosa mimicking bone metastasis.

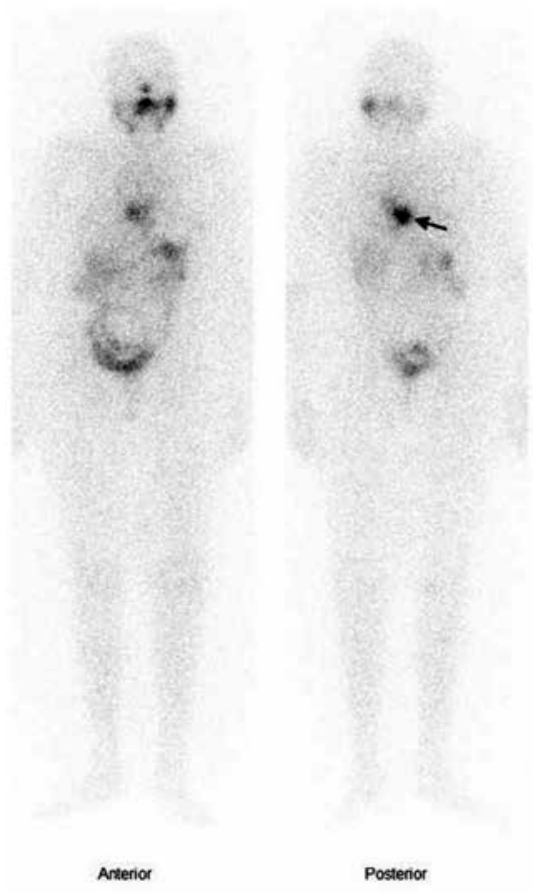

Figure 1. Whole-body iodine-131 scintigraphy. 150 x 252 mm (96 x 96 DPI). 


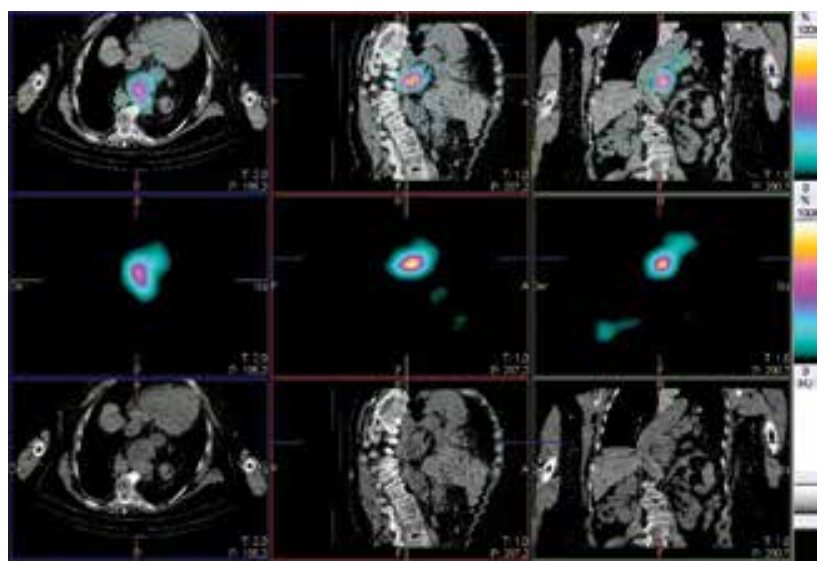

Figure 2. Axial, sagittal and coronal views (left, center and right columns) of hydrib iodine-131 SPECT/CT. Fused images are exposed in the upper row, the SPECT alone in the center row and CT alone in the lower row. $88 \times 60 \mathrm{~mm}(300 \times 300 \mathrm{DPI})$.

\section{DISCUSSION}

The giant diaphragmatic hernia associated with intrathoracic stomach is a very rare entity (1) that seems to be related to increased intra-abdominal pressure and diaphragmatic laxity or kyphoscoliosis deviation in obese patients (2). Usually it corresponds to a type III hiatal hernia, with both sliding and paraesophageal components, wherein at least $30 \%$ of the stomach is in intrathoracic situation (3). Diaphragmatic hernias remain a diagnostic and surgical challenge, in which imaging techniques have become the cornerstone.

In another vein, whole-body ${ }^{131}$ I scintigraphy has proven to be a minimally invasive and safe technique that allows the diagnosis metastasis and recurrence after thyroidectomy when performed at 6-12 months of thyroid remainders ablation with radioiodine, as well as in monitoring during the long-term follow-up. However, the ATA and ETA guidelines state that lowrisk individuals who have had a first post-radioactive iodine remnant ablation whole-body scan with an undetectable $\mathrm{Tg}$ level and a negative anti-Tg antibody level as well as a negative neck ultrasound do not require routine follow-up whole-body scan. Even if false positives in ${ }^{131} \mathrm{I}$ body scans are extremely rare, they have been reported due to contamination by body fluids, ectopic thyroid tissue, infectious and inflammatory processes, benign and malignant tumors, serous cysts and even pulmonary bronchiolitis $(4,5)$. Few cases of false negatives by intrathoracic hernia have been described, because the strange location of the physiological uptake of radioiodine by the gastric mucosa and the limited resolution of planar imaging may suggest that results from a recurrence of the disease (6-10). It can also be seen in healthy tissue, such as thymus, breast, liver and gastrointestinal tract (11) being able to be causes of false positive (12).

In this case, ${ }^{131}$ I SPECT/CT allowed reaching an accurate diagnosis, by discarding unsuspected metastatic bone disease and changing significantly the management and prognosis of the patient. Laparotomy or laparoscopic surgical repair is still the treatment of choice for giant hiatal hernia (13).

\section{Imaging findings}

Whole-body scintigraphy after intravenous injection of $187 \mathrm{MBq}(5 \mathrm{mCi})$ of ${ }^{131} \mathrm{I}$ (Figure 1$)$ demonstrated physiological uptake of the radiotracer as well as a pathological uptake deposit which was located in the midline. This finding was present in both anterior and posterior projections, presenting much greater intensity in the latter (black arrow). Due to lack of precise anatomical definition of this technique, it was compatible with spinal bone metastases as a result of the central location, greater in backplanes and high intensity of uptake.

$\mathrm{A}^{131}$ I SPECT/CT (128x128 matrix; step and shoot mode; 360 degrees orbit; 25 seconds per image; lowdose CT: $140 \mathrm{kV}, 2.5 \mathrm{~mA}$ ) was performed in order to clarifying our clinical and radiological inconsistency. Fused images, SPECT alone and CT alone (top-down, respectively) revealed severe kyphoscoliosis associated with giant diaphragmatic hernia and intrathoracic stomach. In this way, hypermetabolic foci corresponded to physiological radiotracer uptake of the gastric mucosa.

It is known that SPECT/CT was highly accurate in patients who underwent a single challenge of radioiodine therapy (9). In our case, SPECT/CT helped clarify our diagnostic doubt and substantially modified the management and prognosis of the patient.

Disclosure: no potential conflict of interest relevant to this article was reported.

\section{REFERENCES}

1. Grazia KJA, Godoy ZM, Cavallo BI, Cortés AC. Hernia hiatal gigante con estómago intratorácico: Reporte de un caso y revisión de la literatura. Rev Chil Radiol. 2012;18:179-83.

2. Schuchert MJ, Adusumilli PS, Cook CC, Colovos C, Kilic A, Nason $\mathrm{KS}$, et al. The impact of scoliosis among patients with giant paraesophageal hernia. J Gastrointest Surg. 2011;15:23-8.

3. Mitiek MO, Andrade RS. Giant hiatal hernia. Ann Thorac Surg. 2010;89:S2168-73. 
4. García Alonso MP, Balsa Bretón MA, Paniagua Correa C, Castillejos Rodríguez L, Penín González FJ, Elviro Peña R, et al. lodine uptake in the chest in the follow-up of well-differentiated thyroid cancer. Rev Esp Med Nucl. 2011;30:24-8.

5. Thientunyakit T. False-positive 131 l whole-body scan in well-differenciated thyroid cáncer patient with respiratory bronchiolitis. Clin Nucl Med. 2013;38:730-4.

6. HoY, Hicks R. Hiatus hernia: a potential cause of false-positive iodine-131 scan in thyroid carcinoma. Clin Nucl Med. 1998;23:621-2.

7. Schneider JA, Divgi CR, Scott AM, Macapinlac HA, Sonenberg M, Goldsmith SJ, et al. Hiatal hernia on whole-body radioiodine survey mimicking metastatic thyroid cancer. Clin Nucl Med. 1993;18:751-3.

8. Lee YS, Ryu YH, Chang HS, Park CS. Hiatal hernia detected by iodine-131 scan for ablation of thyroid carcinoma. ANZ J Surg. 2010;80:198.
9. Ceylan Gunay E, Erdogan A. Mediastinal radioiodine uptake due to hiatal hernia: a false-positive reason in 131/ scan. Rev Esp Med Nucl. 2010;29:95.

10. Willis $L L$, Cowan RJ. Mediastinal uptake of $\mathrm{I}-131$ in a hiatal hernia mimicking recurrence of papillary thyroid carcinoma. Clin Nucl Med. 1993;18:961-3.

11. Oh JR, Ahn BC. False-positive uptake on radioiodine whole-body scintigraphy: physiologic and pathologic variants unrelated to thyroid cancer. Am J Nucl Med Mol Imaging. 2012;2:362-85.

12. Buton $L$, Morel $O$, Gault $P$, Illouz F, Rodien P, Rohmer V. False-positive iodine-131 whole-body scan findings in patients with differentiated thyroid carcinoma: report of 11 cases and review of the literature. Ann Endocrinol (Paris). 2013;74:221-30.

13. ZhouY, Du H, Che G. Giant congenital diaphragmatic hernia in an adult. J Cardiothorac Surg. 2014;9:31. 